\title{
The strange case of the massive binary HD 149404*
}

\author{
G. Rauw ${ }^{1, \star \star, \ddagger}$, Y. Nazé ${ }^{1, \star \star \star}$, F. Carrier ${ }^{2}$, G. Burki ${ }^{2}$, E. Gosset ${ }^{1, \dagger}, \ddagger$, and J.-M. Vreux ${ }^{1}$ \\ 1 Institut d'Astrophysique et de Géophysique, Université de Liège, 5 avenue de Cointe, 4000 Liège, Belgium \\ 2 Observatoire de Genève, 1290 Sauverny, Switzerland
}

Received 30 October 2000 / Accepted 15 December 2000

\begin{abstract}
We report the analysis of an extensive set of high-resolution spectroscopic observations of the massive binary system HD 149404. We compare different techniques to measure the radial velocities of the heavily blended absorption lines and we derive a new orbital solution. The absorption lines display strong variability that slightly affects the orbital solution and the determination of the spectral types of the components of the binary. We find that the primary is probably of spectral type O7.5 I(f), while the secondary is most likely an ON9.7 I supergiant. The secondary seems to be the most evolved component of the system and its current evolutionary status could best be explained if the system has undergone a Roche lobe overflow episode during the past. The secondary could actually still be rather close to filling its critical volume and this could lead to an enhanced mass loss of the secondary. The spectrum of HD 149404 displays many emission lines some of which show phase-locked line profile variations. In particular, the $\mathrm{H} \alpha$ line displays a double-peaked morphology at orbital phases near conjunction. We investigate the radial velocity behaviour of the emission lines and we find that some of them must be formed in an interaction region. We propose a simple model where some of the optical emission lines arise in a heavily bended shock region.
\end{abstract}

Key words. stars: early-type - binaries: spectroscopic - stars: individual: HD 149404

\section{Introduction}

Over the last decade, substantial progress has been achieved in our understanding of the interaction processes in early-type binary systems. It has been shown that some of the emission lines in the spectra of O-star binaries are at least partially formed in the wind interaction region (e.g. Thaller 1997). Observations of the phase-locked emission line profile variability can be used to constrain the geometry of the interaction region and allow us in principle to distinguish between a colliding wind phenomenon and a Roche lobe overflow (RLOF) process (e.g. Wiggs \& Gies 1993; Rauw et al. 1999). A detailed analysis of the interaction phenomena in binaries in different evolutionary stages is therefore crucial for our understanding of stellar

\footnotetext{
Send offprint requests to: G. Rauw,

e-mail: rauw@astro.ulg.ac.be

* Based on observations collected at the European Southern Observatory (La Silla, Chile) and at the Cerro Tololo InterAmerican Observatory (CTIO).

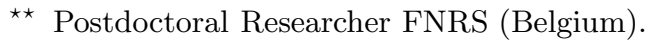

$\star \star \star$ Research Fellow FNRS (Belgium).

$\dagger$ Research Associate FNRS (Belgium).

$\ddagger$ Visiting Astronomer, CTIO, National Optical Astronomy Observatories (NOAO). NOAO is operated by the Association of Universities for Research in Astronomy, Inc. under contract with the National Science Foundation.
}

evolution as well as for a determination of the properties of the stellar winds of the binary components.

In the present paper, we discuss an extensive set of spectra of HD 149404 (= HR 6164 = V 918 Sco). Walborn (1972) assigned an O9 Ia spectral type to the global spectrum of this system. Later on, it was discovered that some lines were actually double, but heavily blended (Conti et al. 1977). A first orbital solution was derived by Massey \& Conti (1979) from a set of optical spectra, and another one was obtained by Stickland \& Koch (1996) from IUE observations (see Table 4). Several spectral features of HD 149404 point towards an interaction between the two components. In fact, Massey \& Conti (1979) noticed the double-peaked structure of the $\mathrm{H} \alpha$ emission profile in the spectrum of HD 149404 and they interpreted this feature as a signature of an ongoing Roche lobe overflow process. More recently, Thaller (1998) suggested that the behaviour of the two $\mathrm{H} \alpha$ peaks was due to a colliding wind interaction. HD 149404 is also known to display the so-called "Struve-Sahade effect" (Stickland 1997). So far, the origin of this effect remains unclear although Gies et al. (1997) suggest that it could result from photospheric heating by the X-ray emission generated in the wind interaction region.

This paper is organized as follows: the observations and the data reduction are presented in Sect. 2 and the 


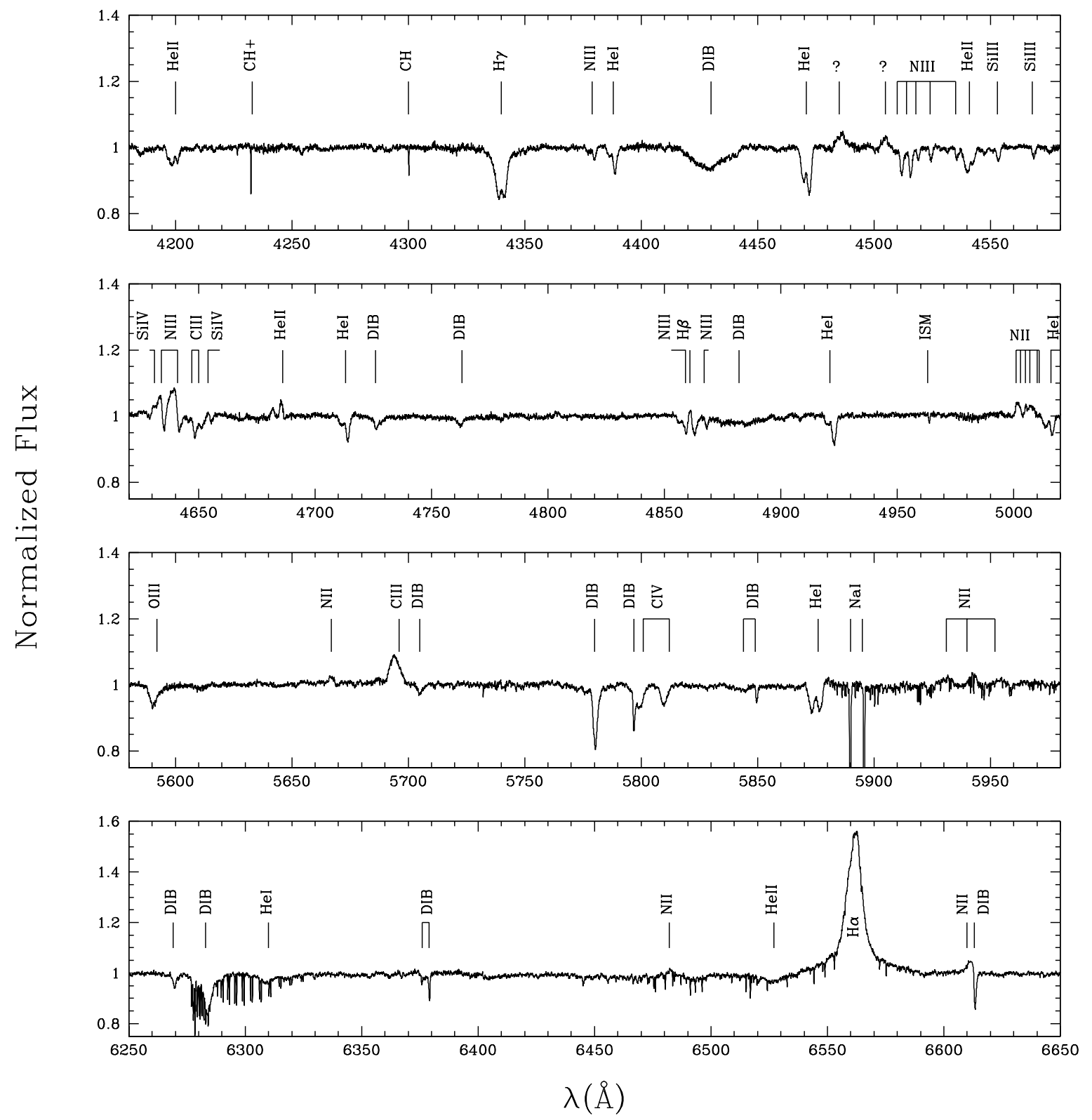

Fig. 1. Spectrum of HD 149404 as observed with the FEROS spectrograph on HJD $2451299.800(\phi=0.23)$. At this orbital phase, the spectrum of the most massive star is shifted towards the blue

spectral features of HD 149404 are discussed in Sect. 3 . Different techniques are used to measure the radial velocities of the absorption lines and a new orbital solution is derived in Sect. 4. In Sect. 5, we discuss the luminosity ratio of the binary components. As a next step, we examine the variations of the emission lines (Sect.6). Finally, we discuss a possible model for the interaction in HD 149404 as well as the evolutionary status of the system (Sect.7).

\section{Observations and data reduction}

We observed HD 149404 over a period of 3 years with various instruments. A first set of spectra, covering roughly $45 \AA$ centred on the HeI $\lambda 4471$, He II $\lambda 4686$ and N III $\lambda \lambda 4634-41$ lines, was obtained in June 1997 with the 1.4 m Coudé Auxiliary Telescope (CAT) at ESO, La Silla, feeding the Coudé Echelle Spectrometer (CES) and the Long Camera (LC). Additional data (covering $20 \AA$ around the HeI $\lambda 4471$ and He II $\lambda 4686$ lines) were taken with the same spectrograph equipped with the Very Long Camera (VLC) in April-May 1998 and July 1998. In all cases, the detector was ESO CCD\#38, a Loral $2688 \times 512$ CCD with pixel size $15 \mu \mathrm{m} \times 15 \mu \mathrm{m}$. The nominal resolving power was 70000-80000. Typical exposure times were of the order of $15 \mathrm{~min}$ and the average signal-to-noise ratio was about 150 .

In May 1999 and May 2000, we observed HD 149404 with the Fiber-fed Extended Range Optical Spectrograph (FEROS, Kaufer et al. 1999) at the ESO $1.52 \mathrm{~m}$ telescope at La Silla. The spectra cover the wavelength range 3650$9200 \AA$ with a resolving power of 48000 . The detector was a $2 \mathrm{k} \times 4 \mathrm{k}$ EEV CCD with pixel size $15 \mu \mathrm{m} \times 15 \mu \mathrm{m}$. 
The typical exposure time was $2 \mathrm{~min}$ and the $S / N$ ratio at $6000 \AA$ is about 160 .

Five more spectra were taken in May-June 1999 with the Bench-Mounted Echelle spectrograph (BME) fed by the $1.5 \mathrm{~m}$ Ritchey-Chrétien telescope at CTIO. These data covered the spectral range $3750-5800 \AA$. The typical $S / N$ ratio was 120 at $4620 \AA$ for exposure times of about $20 \mathrm{~min}$. The detector used was a TEK CCD with $2048 \times 2048$ pixels of $24 \mu \mathrm{m} \times 24 \mu \mathrm{m}$ and the resolving power was 45000 .

Finally, twelve echelle spectra were obtained in February 2000 with the $1.2 \mathrm{~m}$ Euler Swiss telescope at La Silla, equipped with the Coralie spectrograph and a $2 \mathrm{k} \times 2 \mathrm{k}$ CCD with pixel size $15 \mu \mathrm{m} \times 15 \mu \mathrm{m}$. The Coralie instrument is an improved version of the Elodie spectrograph (Baranne et al. 1996). These observations covered the spectral range $3875-6800 \AA$ with a resolving power of 50000 . The integration times varied between 30 and $45 \mathrm{~min}$ and the typical $S / N$ ratio at $5000 \AA$ was $120-150$.

The CAT data were reduced in a standard way with the MIDAS software developed at ESO. The spectra were normalized by fitting a low order polynomial to the continuum. The FEROS spectra were reduced using the appropriate context of the MIDAS environment together with several dedicated routines kindly provided by Dr. O. Stahl. The extracted FEROS spectra were rectified by fitting a polynomial of degree 4 or 5 to the continuum over a range of a few hundred $\AA$ at once.

The CTIO data were reduced using the IRAF ${ }^{1}$ package, following the recommendations of the BME User's Manual. We used the smoothed projector flat field exposures as a first guess of the continuum, and the spectra were finally normalized using a low-order polynomial.

The Coralie data were reduced at the telescope, using the INTER-TACOS (INTERpreter for the Treatment, the Analysis and the COrrelation of Spectra) software developed by D. Queloz and L. Weber at the Geneva Observatory (Baranne et al. 1996). Next, the normalisation was done by dividing by the blaze fits and finally by fitting a polynomial to the continuum.

Whenever several exposures were available for the same night, we averaged them to increase the $S / N$ ratio. Some preliminary results based on our data were already presented by Nazé et al. (2000).

\section{The spectrum of HD 149404}

A FEROS spectrum of HD 149404 is shown in Fig. 1. Besides the lines of the upper Balmer series (above $\mathrm{H} \gamma$ ), the spectrum reveals many typical O-star absorption lines of He I, He II, N III, Si III, Si IV, C IV and O III. The less massive star exhibits strong N III absorptions whereas N III is seen as a weak absorption or emission ( $\mathrm{N}$ III $\lambda \lambda 4634$ $41)$ in the primary's spectrum. The spectrum reveals also a number of diffuse interstellar bands (DIBs), as well as

\footnotetext{
${ }^{1}$ IRAF is distributed by the National Optical Astronomy Observatories.
}

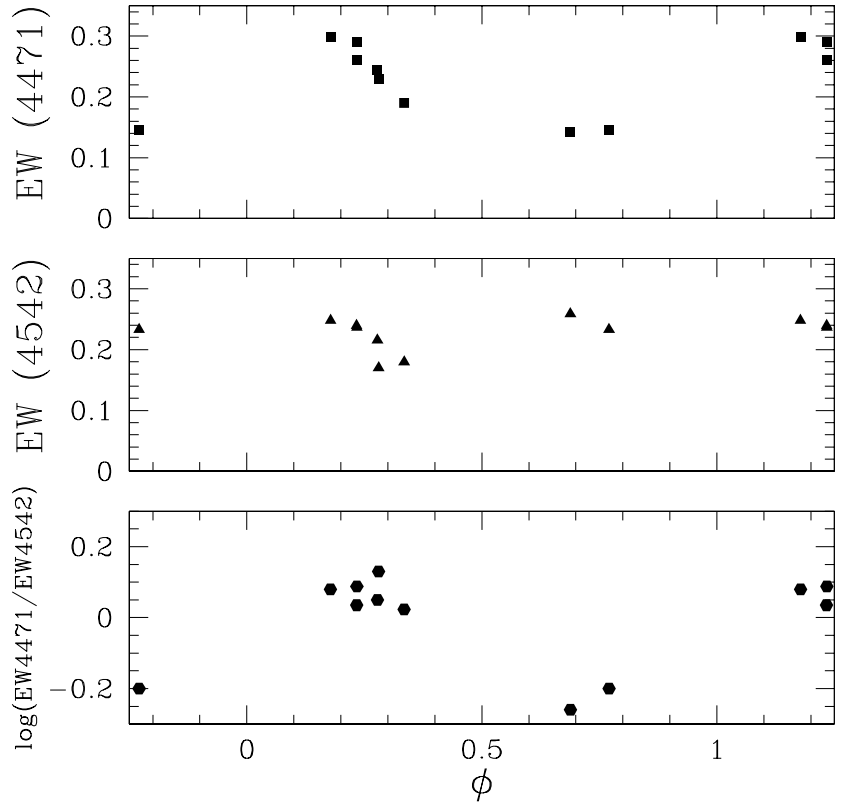

Fig. 2. Variations of the equivalent width of the He I $\lambda 4471$ and He II $\lambda 4542$ lines and of the $\log \left(W^{\prime}\right)$ criterion for the primary star as a function of orbital phase

interstellar absorptions due to $\mathrm{NaI}, \mathrm{Ca} I \mathrm{I}, \mathrm{CH}$ and $\mathrm{CH}^{+}$. However, the most remarkable feature of HD 149404 is the important number of emission lines in its spectrum: $\mathrm{H} \alpha$, $\mathrm{H} \beta$, He II $\lambda 4686$, C III $\lambda 5696$, as well as several N II and Si IV lines. We will come back to some of these features in the next sections. Throughout this paper we will refer to the more massive star as the primary.

\subsection{Spectral types}

Only the least blended spectra are suitable for the spectral classification. Following Conti \& Alschuler (1971), Conti \& Frost (1977) and Mathys $(1988,1989)$, we adopt the $\log W^{\prime}=\log (E W(4471) / E W(4542))$ classification criteria. We caution that the equivalent width $(E W)$ of the He II $\lambda 4542$ line of the individual components is rather difficult to measure in the case of HD 149404 because of the severe blending.

For the secondary, we find $\log W^{\prime}$ between 0.51 and 0.83 , corresponding to a spectral type O9.7 (using the Mathys criteria). The 09.7 classification is also supported by the fact that the He II $\lambda 4542$ and Si III $\lambda 4552$ absorptions have about the same strength (Walborn \& Fitzpatrick 1990).

For the primary, we obtain $\log W^{\prime}$ between -0.26 and 0.13. More specifically, we find an O6.5 spectral type for orbital phases near 0.75 , i.e. when the primary is moving towards us (see Fig. 2), and an O7.5 type half a period later. In fact, Fig. 2 indicates that the orbital variation of $\log W^{\prime}$ is essentially due to the variation of the equivalent width of the He I $\lambda 4471$ line. It is interesting to note that the He I $\lambda 4471$ absorption of the secondary displays 


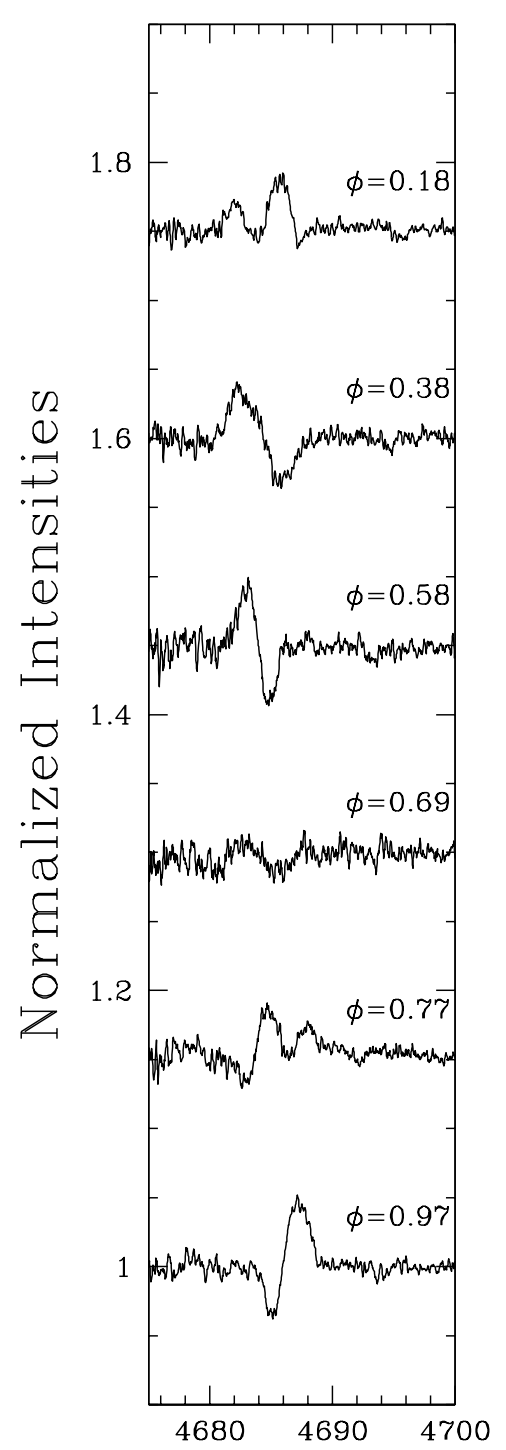

He II $\lambda 4686 \AA$

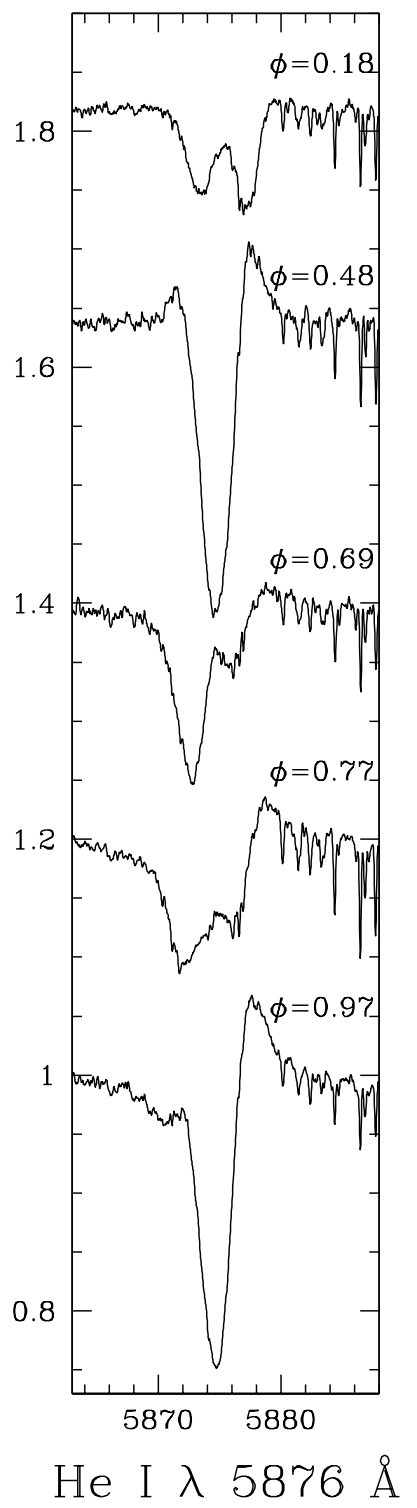

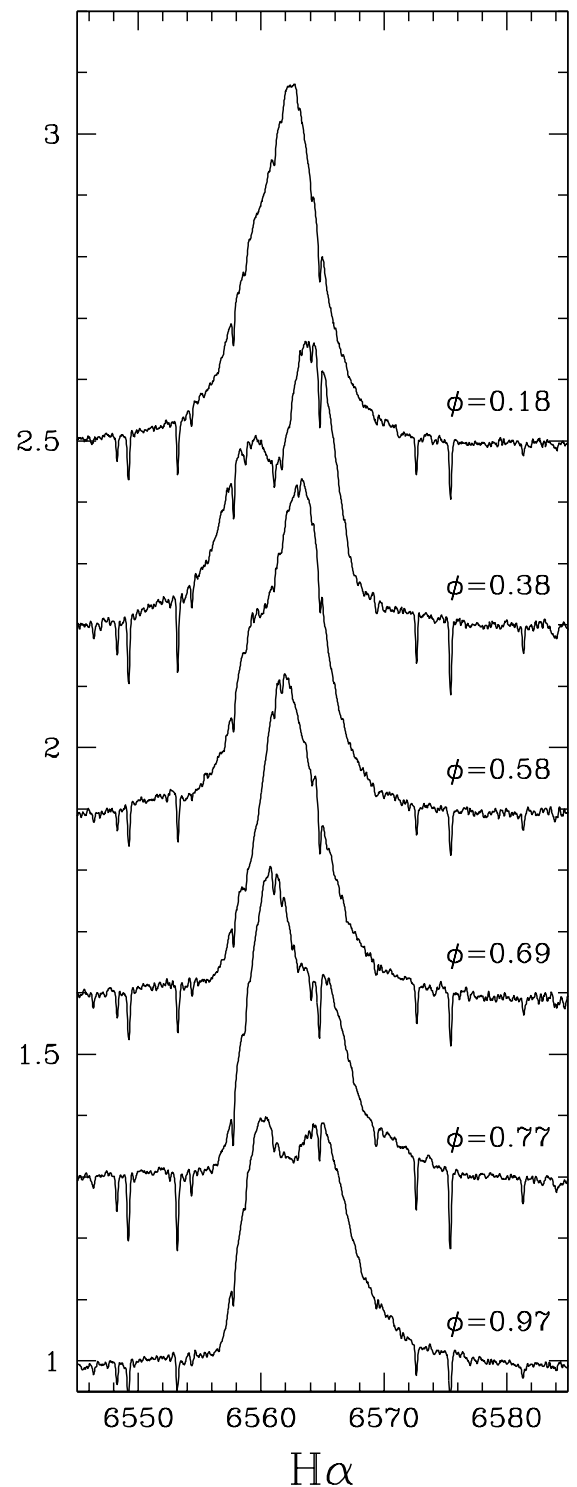

Fig. 3. Variation of the He II $\lambda 4686$, He I $\lambda 5876$ and $\mathrm{H} \alpha$ lines with phase

the opposite behaviour ${ }^{2}$, i.e. it appears stronger around $\phi=0.75$ and weaker near $\phi=0.25$ (Nazé et al. 2000). The behaviour of both absorption components at He I $\lambda 4471$ could therefore at least partially reflect the blending with a weak, slightly red-shifted emission component that does not follow the orbital motion of neither star. Such an emission feature is indeed seen in the He I $\lambda 5876$ line (see Fig. 3) and we actually detect a weak emission on some of the He I $\lambda 4471$ spectra taken near conjunction. At phases near 0.75 , the emission is blended with the primary's absorption, hence reducing its equivalent width. The reverse situation occurs half a cycle later, when the secondary's absorption is blended with the emission. As the primary's $E W$ is most probably less affected by the emission feature

\footnotetext{
${ }^{2}$ We caution however that the variation of the $\log W^{\prime}$ criterion of the secondary results also from variations of the $E W$ of the HeII $\lambda 4542$ line that are more pronounced than for the primary.
}

around $\phi=0.25$, we adopt the O7.5 classification for this star (see also Nazé et al. 2000).

For the luminosity classification, we rely on the $\log W^{\prime \prime}=\log (E W(\operatorname{Si} I V \lambda 4089) / E W($ He I $\lambda 4143))$ criterion introduced by Conti \& Alschuler (1971). This turned out to be even more difficult than the spectral classification. In fact, He I $\lambda 4143$ could be deblended on a few spectra only and we could only set an upper limit of $\leq 0.07 \AA$ on the $E W$ of the primary's He I $\lambda 4143$ line. For the secondary, we find $\log W^{\prime \prime}$ between 0.36 and 0.65 corresponding to a supergiant classification. In the same way, our upper limit on the primary's $E W($ He I $\lambda 4143)$ yields $\log W^{\prime \prime} \geq 0.4$ (at all orbital phases), also corresponding to luminosity class I. We caution that a similar effect as for the He I $\lambda 4471$ line could alter the $E W$ s of the He I $\lambda 4143$ lines, although we do not expect this effect to significantly affect our conclusions.

On the other hand, He II $\lambda 4686$ could be in absorption in the spectrum of both stars (see below), an 
Table 1. Radial velocities (in $\mathrm{km} \mathrm{s}^{-1}$ ) of the most prominent interstellar lines in the spectrum of HD 149404. The quoted uncertainties correspond to the 1- $\sigma$ dispersion of the $R V$ s measured during the same observing run

\begin{tabular}{lccc}
\hline \multicolumn{1}{c}{ Line } & FEROS 1999 & Coralie & FEROS 2000 \\
\hline \hline Ca II $\lambda 3933$ & $-3.9 \pm 0.2$ & $-4.5 \pm 0.3$ & $-2.7 \pm 0.1$ \\
Ca II $\lambda 3968$ & $-3.8 \pm 0.3$ & $-5.3 \pm 0.5$ & $-3.8 \pm 0.1$ \\
$\mathrm{CH}^{+} \lambda 3958$ & $-2.4 \pm 0.6$ & $-3.6 \pm 2.9$ & $-2.3 \pm 0.8$ \\
$\mathrm{CH}^{+} \lambda 4233$ & $-1.6 \pm 0.6$ & $-2.7 \pm 0.4$ & $-1.9 \pm 0.4$ \\
$\mathrm{CH} \lambda 4300$ & $-0.4 \pm 0.2$ & $-1.0 \pm 0.3$ & $-0.4 \pm 0.4$ \\
$\mathrm{Na}$ I $\lambda 5890$ & $-7.8 \pm 0.3$ & $-8.4 \pm 0.4$ & $-8.2 \pm 0.0$ \\
$\mathrm{Na}$ I $\lambda 5896$ & $-7.9 \pm 0.3$ & $-8.2 \pm 0.3$ & $-7.9 \pm 0.0$ \\
\hline
\end{tabular}

unlikely feature for luminosity class I stars, especially for Ia stars (Walborn 1971). Finally, the N III $\lambda \lambda 4634-41$ emission lines appear to follow more or less the motion of the primary. Therefore we add an (f) tag to the spectral classification of the primary.

\subsection{Interstellar lines}

We have measured the radial velocities $(R V \mathrm{~s})$ of the most prominent interstellar lines (Table 1). Both Ca II lines exhibit a blueshifted much fainter component which is only visible on the FEROS spectra.

We emphasize the very good night-to-night stability of the FEROS and Coralie spectrographs as revealed by the small $1-\sigma$ dispersions of the $R V \mathrm{~s}$ in Table 1 . There is a slight difference of the order of $0.5 \mathrm{~km} \mathrm{~s}^{-1}$ between the $R V$ s derived from the FEROS and the Coralie data. Moreover, we notice that there is a slight $R V$-difference between the Ca II and Na I lines. Stickland \& Koch (1996) adopted a radial velocity of $-7.5 \mathrm{~km} \mathrm{~s}^{-1}$ for the interstellar features in their IUE spectra, which is consistent with our results for the $\mathrm{Na}$ I lines.

\section{Orbital solution}

\subsection{Radial velocity determination}

Since He I $\lambda 4471$ is the most extensively covered absorption line in our data set and since it is also usually the least blended one, we decided to use the $R V \mathrm{~s}$ of this line to determine a new orbital solution. Other lines are either weak (e.g. He II $\lambda 4542$ ), blended with other weaker absorptions (He II $\lambda 4200$ blended with N III $\lambda 4196$ ) or affected by a rather strong emission component (He I $\lambda 5876$ ). These features are thus not well suited to measure the $R V \mathrm{~s}$ of the binary components.

We notice that the intensities of the absorption lines (including He I $\lambda 4471$, see Fig. 2 and Nazé et al. 2000) change with phase. This phenomenon is not unexpected since HD 149404 is known to display variations of the line strength in the UV that could be related to the so-called "Struve-Sahade effect" (Stickland 1997).

The radial velocities were determined by fitting two Gaussians whenever it was possible to do so. However, the lines remain heavily blended over the main part of the orbit and even around quadrature the lines are not completely separated. Therefore, two different techniques were tested to determine the radial velocities when Gaussian fitting turned out to be impossible. In both cases we use a template line profile built from the spectra at maximum separation.

(1) When the lines are partially blended, the "outer" wing of the strongest line is rather unaffected by the other line. Fitting the corresponding wing of the template spectrum to the observed profile yields the position of the strongest line. Next, the template profile of the strongest line is shifted to the correct position and subtracted from the observed spectrum. The position of the weaker line is finally obtained by fitting a Gaussian to the residuals;

(2) Alternatively we have attempted a simultaneous fit of the positions of the lines of both components. To this aim we have shifted both templates independently and simultaneously until we found a best fit to the observed profile.

The resulting radial velocities are presented in Table 2.

Given the difficulties due to the severe blending of the lines, we also used another method developed at the Geneva observatory to determine the radial velocities. The velocities are obtained by cross-correlation of the FEROS and Coralie spectra with a mask built from a synthetic spectrum. This method is well known for less massive stars, but this is the first attempt to extend it to the optical spectra of O-type stars. A synthetic O-star spectrum in the 3875-6820 A spectral domain was generated using the SYNSPEC (Hubeny et al. 1994) code with the model atmospheres interpolated from the Kurucz ATLAS9 (1994) grid. The Vienna Atomic Line Database was used to create a line list for the spectrum synthesis (Kupka et al. 1999). The spectrum synthesis programme uses an LTEmodel which is certainly not appropriate to determine the properties of O-stars, but should be sufficient for the radial velocity determinations. Many tests were conducted and eventually, a synthetic spectrum with $T_{\text {eff }}=42500 \mathrm{~K}$ was adopted because it provided the most narrow crosscorrelation peak. Blended lines as well as emission lines or lines that occur in the synthetic spectrum but are absent from the actual spectrum were removed from the mask. Therefore, the final mask no longer corresponds to a single $T_{\text {eff }}$ but should be valid over a rather broad temperature range from about 35000 to $42500 \mathrm{~K}$. The radial velocities were finally obtained from the cross-correlation function by fitting two Gaussians. The results are presented in the last two columns of Table 2 .

\subsection{Period determination}

We used the generalized Fourier spectrogramme technique of Heck et al. (1985, hereafter HMM, see their Eq. (1)) and 
Table 2. Barycentric radial velocities obtained for He I $\lambda 4471$ and by a correlation with a synthetic mask. The dates of the observations are given in the format HJD-2450000 and the orbital phases are those corresponding to the circular orbital solution derived from the cross-correlation $R V$ s (see Table 3 ). The method used to derive the $R V$ s of the He I $\lambda 4471$ line is specified in the sixth column: $d$ (nbr of Gaussians) = Gaussian fit, $c 1(c 2)=1$ st (2nd) technique (see text), $m=$ mean of techniques listed in brackets

\begin{tabular}{|c|c|c|c|c|c|c|c|}
\hline \multirow[t]{2}{*}{ Instr. } & \multirow[t]{2}{*}{ Date } & \multirow[t]{2}{*}{$\phi$} & \multicolumn{3}{|c|}{ He I $\lambda 4471$} & \multicolumn{2}{|c|}{ cross-correlation } \\
\hline & & & $\begin{array}{r}R V_{1} \\
\mathrm{~km} \mathrm{~s}^{-1} \\
\end{array}$ & $\begin{array}{r}R V_{2} \\
\mathrm{~km} \mathrm{~s}^{-1} \\
\end{array}$ & Method & $\begin{array}{r}R V_{1} \\
\mathrm{~km} \mathrm{~s}^{-1} \\
\end{array}$ & $\begin{array}{r}R V_{2} \\
\mathrm{~km} \mathrm{~s}^{-1} \\
\end{array}$ \\
\hline$\overline{\mathrm{LC}}$ & 622.588 & 0.23 & -107.0 & 771.7 & $\overline{d(2)}$ & & \\
\hline $\mathrm{LC}$ & 623.595 & 0.34 & -107.1 & 35.5 & $m(c 1, c 2)$ & & \\
\hline $\mathrm{LC}$ & 624.552 & 0.43 & -86.9 & -14.2 & $m(c 1, c 2)$ & & \\
\hline $\mathrm{LC}$ & 625.593 & 0.54 & -58.2 & -58.2 & $d(1)$ & & \\
\hline $\mathrm{LC}$ & 626.556 & 0.64 & -12.3 & -109.4 & $c 1$ & & \\
\hline VLC & 925.944 & 0.14 & -103.4 & 26.8 & $m(d(2), c 1)$ & & \\
\hline VLC & 926.944 & 0.24 & -124.2 & 47.6 & $d(2)$ & & \\
\hline VLC & 930.884 & 0.65 & 0.2 & -136.9 & $m(d(2), c 1)$ & & \\
\hline VLC & 932.946 & 0.86 & -21.2 & -124.6 & $m(d(2), c 1)$ & & \\
\hline VLC & 937.914 & 0.36 & -111.9 & 13.7 & $m(d(2), c 1)$ & & \\
\hline VLC & 995.635 & 0.24 & -116.6 & 72.5 & $d(2)$ & & \\
\hline VLC & 996.565 & 0.34 & -96.9 & 31.3 & $m(c 1, c 2)$ & & \\
\hline VLC & 997.581 & 0.44 & -66.3 & 4.1 & $m(c 1, c 2)$ & & \\
\hline VLC & 998.572 & 0.54 & -73.9 & -73.9 & $d(1)$ & & \\
\hline VLC & 999.572 & 0.64 & -48.5 & -147.4 & $m(c 1, c 2)$ & & \\
\hline VLC & 1000.544 & 0.74 & 36.7 & -160.6 & $d(2)$ & & \\
\hline FER. & 1299.800 & 0.23 & -117.8 & 52.9 & $d(2)$ & -108.0 & 55.5 \\
\hline FER. & 1300.793 & 0.34 & -118.8 & 43.5 & $d(2)$ & -109.0 & 49.7 \\
\hline FER. & 1301.797 & 0.44 & -79.6 & -23.6 & $c 2$ & -67.1 & 3.0 \\
\hline FER. & 1302.788 & 0.54 & -67.5 & -67.5 & $d(1)$ & -39.2 & -70.5 \\
\hline FER. & 1304.796 & 0.74 & 28.5 & -127.6 & $d(2)$ & 12.4 & -138.5 \\
\hline FER. & 1323.741 & 0.67 & 43.8 & -119.7 & $d(2)$ & 15.4 & -124.0 \\
\hline FER. & 1327.766 & 0.08 & -77.6 & 4.6 & $c 2$ & -75.0 & 2.5 \\
\hline BME & 1328.700 & 0.18 & -135.5 & -0.5 & $c 2$ & & \\
\hline BME & 1329.705 & 0.28 & -131.3 & 46.0 & $d(2)$ & & \\
\hline BME & 1330.707 & 0.38 & -92.9 & 0.6 & $c 2$ & & \\
\hline BME & 1331.717 & 0.49 & -52.5 & -52.5 & $d(1)$ & & \\
\hline BME & 1332.728 & 0.59 & -32.8 & -120.5 & $c 2$ & & \\
\hline COR. & 1578.866 & 0.67 & 38.8 & -115.1 & $d(2)$ & 6.8 & -123.1 \\
\hline COR. & 1579.879 & 0.77 & 38.0 & -139.7 & $d(2)$ & 18.8 & -146.6 \\
\hline COR. & 1580.872 & 0.87 & -45.5 & -122.1 & $c 2$ & -9.6 & -111.9 \\
\hline COR. & 1581.864 & 0.97 & -58.8 & -58.8 & $d(1)$ & -34.2 & -64.0 \\
\hline COR. & 1582.869 & 0.08 & -70.5 & 11.2 & $d(2)$ & -80.6 & -3.7 \\
\hline COR. & 1583.877 & 0.18 & -92.8 & 63.5 & $d(2)$ & -98.0 & 54.7 \\
\hline COR. & 1584.857 & 0.28 & -101.0 & 65.9 & $d(2)$ & -99.7 & 58.1 \\
\hline COR. & 1585.845 & 0.38 & -84.4 & 8.9 & $d(2)$ & -85.9 & 8.2 \\
\hline COR. & 1586.870 & 0.48 & -46.6 & -46.6 & $d(1)$ & -65.0 & -30.9 \\
\hline COR. & 1587.861 & 0.58 & -48.2 & -104.7 & $c 2$ & -10.0 & -89.8 \\
\hline COR. & 1588.886 & 0.69 & 31.8 & -133.8 & $d(2)$ & 8.1 & -132.9 \\
\hline COR. & 1590.886 & 0.89 & -49.8 & -108.3 & $c 2$ & -9.1 & -98.5 \\
\hline FER. & 1668.892 & 0.84 & -44.2 & -150.0 & $c 2$ & -4.4 & -127.2 \\
\hline FER. & 1669.769 & 0.93 & -73.5 & -73.5 & $d(1)$ & -18.1 & -82.2 \\
\hline FER. & 1670.770 & 0.03 & -46.1 & -46.1 & $d(1)$ & -61.0 & -15.0 \\
\hline FER. & 1671.775 & 0.13 & -95.3 & 27.8 & $d(2)$ & -85.5 & 27.9 \\
\hline FER. & 1672.766 & 0.23 & -104.6 & 67.4 & $d(2)$ & -102.5 & 64.8 \\
\hline FER. & 1673.895 & 0.35 & -106.7 & 18.8 & $d(2)$ & -89.6 & 33.7 \\
\hline
\end{tabular}

the methods of Renson (1978) and Lafler \& Kinman (1965, hereafter LK) to determine an improved value of the orbital period. To this aim, we applied the period search techniques to the time series of the $R V_{1}-R V_{2}$ radial velocity differences as determined from the He I $\lambda 4471$ line in our data. To improve the accuracy of the result, we included also the data of Stickland \& Koch (1996). The HMM method yields an orbital period of 9.81475 days (in agreement with the results of the Renson method) whilst the LK method yields 9.81431 days. The estimation of 
the error on a period is not a straightforward problem. A secure upper limit on the error can be estimated from the width of the peak (which regardless of the method is of the order $1 / \Delta T$ where $\Delta T$ is the total time spanned by the data set) i.e. 0.014 day in the present case. Empirically, the error is usually a substantial fraction of it. Under the hypothesis that the data are well distributed over $\Delta T$ and that the sampling does not suffer from any particular problem, we can use Eq. (20) of Lucy \& Sweeney (1971) to compute a formal error. In the present case this yields $\sigma_{\mathrm{P}}=0.00084$ day. The three methods hereabove yield values of the period that overlap within the errors and in the following we will adopt the period obtained with the HMM technique.

\subsection{Orbital elements}

We used our radial velocity measurements to determine a new orbital solution. As a first step, we considered the possibility of an eccentric solution. The $R V \mathrm{~s}$ determined by cross-correlation of our echelle spectra with a mask yield a very small eccentricity of $e=0.02 \pm 0.02$. We notice that the $R V \mathrm{~s}$ of the He I $\lambda 4471$ line alone yield a larger value of $e=0.20 \pm 0.05$, but this result is most probably due to the systematic deviation of the He I $\lambda 4471 R V \mathrm{~s}$ at certain orbital phases with respect to the $R V \mathrm{~s}$ derived by cross-correlation. In the following, we will focus on the non-eccentric solutions. The orbital elements determined using the various datasets are listed in Table 3 and the curves shown in Figs. 4 and 5.

A simple inspection of the solutions listed in Table 3 shows that most of the orbital elements overlap within their errors. The errors on the cross-correlation solution are however much smaller and the data points show little scatter around the best fit solution. This illustrates the power of the mask cross-correlation technique to handle the orbital solutions of heavily blended early-type binaries and, in the following, we will use the orbital elements determined from this solution. The orbital phases used throughout this paper refer to the ephemerides from this latter solution.

The $R V$ s determined for the He I $\lambda 4471$ line are usually in rough agreement with those determined by mask cross-correlation if we account for the slight difference in the $\gamma$ velocities. A major disagreement is apparent around $\phi \sim 0.75$ for the $R V \mathrm{~s}$ of the primary star that cluster systematically some $40 \mathrm{~km} \mathrm{~s}^{-1}$ above the best fit curve (Fig. 4). It is interesting to note that the most discrepant He I $\lambda 4471 R V$ s occur precisely in the range of orbital phases when the primary's lines display a reduced $E W$. The discrepant $R V \mathrm{~s}$ of the primary near $\phi \sim 0.75$ lead to a somewhat larger mass ratio inferred from the $R V \mathrm{~s}$ of the He I $\lambda 4471$ line than from the cross-correlation $R V \mathrm{~s}$. In this context, we note that an orbital solution derived from the $R V \mathrm{~s}$ of the HeII $\lambda 5412$ line yields a mass ratio of 0.619 (Nazé et al. 2000), in better agreement with the value obtained from the cross-correlation method.
Our orbital solutions yield apparent systemic velocities for the two components that are somewhat different with the primary showing the more negative $\gamma$. This could indicate that the primary's wind is slightly stronger than that of the secondary. However, the effect is much smaller for HD 149404 than for some other evolved early-type binaries (e.g. Rauw et al. 1999, 2000b).

Table 4 provides an overview of the orbital solutions of HD 149404 available in the literature. We notice that the mass ratio determined from our solution is intermediary between the values proposed by Massey \& Conti (1979) and by Stickland \& Koch (1996). Several authors argue that the actual mass ratio might be close to unity. In fact, Massey \& Conti (1979) suggested a value of $q \sim 1$ based on the $R V \mathrm{~s}$ of the $\mathrm{N}$ III $\lambda \lambda 4634-41$ emission lines which they attributed to the primary star. Though it has been shown that the N III emissions might actually form in the photosphere of Of-type stars (Mihalas \& Hummer 1973), our observations of HD 149404 reveal a rather complex behaviour of these lines that is not consistent with a purely photospheric origin (see below). A mass ratio near unity was also suggested by Hutchings \& van Heteren (1981) based on a preliminary analysis of four IUE spectra and by Penny et al. (1996) from a comparison of the location of the components of HD 149404 in the H-R diagram with theoretical evolutionary tracks of single stars. It is worth mentioning that all the prominent absorption lines in the spectrum of HD 149404 (He II $\lambda 4542$, He I $\lambda 4713$, He I $\lambda 4921, \mathrm{~N}$ III $\lambda \lambda 4510-24, \ldots$ ) yield a mass ratio in agreement with our orbital solution.

Finally, we note that including the Stickland \& Koch (1996) data in our orbital solution yields orbital elements very similar to those found from our data alone.

\subsection{Inclination}

Massey \& Conti (1979) report a photometric study of HD 149404 by Dr. N. Morrison that revealed some variability at a rather low level $(0.03 \mathrm{mag})$. The lightcurve could probably be explained by ellipsoidal variations but there are not enough data points to draw any firm conclusion (Morrison 2000).

Luna (1988) obtained polarimetric observations of HD 149404 that yield only an upper limit of $50^{\circ}$ on the orbital inclination. Vanbeveren \& de Loore (1980) propose an inclination of $28^{\circ}$ based on the orbital solution of Massey \& Conti (1979) and on a comparison of the primary parameters with typical masses of O7 stars. Penny et al. (1996) derived an upper limit of $31^{\circ}$ from the condition that neither of the two stars should fill its Roche lobe, and a lower limit of $15^{\circ}$ by setting an upper limit of $150 M_{\odot}$ on the primary's mass.

Comparing our minimum masses from Table 3 with "typical" masses of O9.7 I and O7.5 I supergiants (e.g. Howarth \& Prinja 1989), we find an inclination of $20.3^{\circ}$ for the primary and $21.0^{\circ}$ for the secondary. Adopting $21^{\circ}$ as a "realistic" estimate, the Roche lobe radii are equal to 
Table 3. Orbital solutions adopting a period of $P=9.81475$ days and assuming $e=0.0 . T_{0}$ corresponds to the conjunction with the secondary in front. The quoted errors correspond to $1 \sigma$ uncertainties

\begin{tabular}{|c|c|c|c|c|}
\hline & \multicolumn{2}{|c|}{ He I $\lambda 4471$} & \multicolumn{2}{|c|}{ cross-correlation } \\
\hline & Prim. & Second. & Prim. & Second. \\
\hline$\overline{T_{0}(\mathrm{HJD}-2450000)}$ & \multicolumn{2}{|c|}{$1680.188 \pm 0.531$} & \multicolumn{2}{|c|}{$1680.279 \pm 0.174$} \\
\hline$\gamma\left(\mathrm{km} \mathrm{s}^{-1}\right)$ & $-54.0 \pm 5.2$ & $-46.4 \pm 11.5$ & $-46.6 \pm 1.7$ & $-41.6 \pm 3.5$ \\
\hline$K\left(\mathrm{~km} \mathrm{~s}^{-1}\right)$ & $62.8 \pm 6.0$ & $99.1 \pm 9.0$ & $59.7 \pm 2.0$ & $98.7 \pm 3.2$ \\
\hline$a \sin i\left(R_{\odot}\right)$ & $12.2 \pm 1.2$ & $19.2 \pm 1.9$ & $11.6 \pm 0.4$ & $19.1 \pm 0.6$ \\
\hline$m \sin ^{3} i\left(M_{\odot}\right)$ & $2.64 \pm 0.66$ & $1.68 \pm 0.42$ & $2.52 \pm 0.21$ & $1.52 \pm 0.13$ \\
\hline$q=m_{2} / m_{1}$ & \multicolumn{2}{|c|}{$0.634 \pm 0.085$} & \multicolumn{2}{|c|}{$0.605 \pm 0.027$} \\
\hline$R_{\mathrm{RL}} /\left(a_{1}+a_{2}\right)$ & $0.42 \pm 0.01$ & $0.34 \pm 0.01$ & $0.42 \pm 0.01$ & $0.34 \pm 0.01$ \\
\hline$R_{\mathrm{RL}} \sin i\left(R_{\odot}\right)$ & 13.2 & 10.7 & 13.0 & 10.3 \\
\hline $\operatorname{rms}(\mathrm{O}-\mathrm{C})\left(\mathrm{km} \mathrm{s}^{-1}\right)$ & \multicolumn{2}{|c|}{18.3} & \multicolumn{2}{|c|}{6.1} \\
\hline
\end{tabular}

Table 4. Orbital solutions from the literature. $T_{0}$ corresponds to the conjunction with the less massive star in front

\begin{tabular}{|c|c|c|c|c|}
\hline & \multicolumn{2}{|c|}{ Massey \& Conti } & \multicolumn{2}{|c|}{ Stickland \& Koch } \\
\hline & Prim. & Second. & Prim. & Second. \\
\hline$P$ (days) & \multicolumn{2}{|c|}{9.813} & \multicolumn{2}{|c|}{9.81452} \\
\hline$T_{0}(\mathrm{HJD}-2440000)$ & \multicolumn{2}{|c|}{2503.607} & \multicolumn{2}{|c|}{4446.849} \\
\hline$e$ & \multicolumn{2}{|c|}{0 (adopted) } & \multicolumn{2}{|c|}{0 (adopted) } \\
\hline$\gamma\left(\mathrm{km} \mathrm{s}^{-1}\right)$ & -28.0 & -37.0 & -60.0 & -52.5 \\
\hline$K\left(\mathrm{~km} \mathrm{~s}^{-1}\right)$ & 60.0 & 101.0 & 64.3 & 98.1 \\
\hline$a \sin i\left(R_{\odot}\right)$ & 11.60 & 19.63 & 12.47 & 19.04 \\
\hline$m \sin ^{3} i\left(M_{\odot}\right)$ & 2.7 & 1.6 & 2.64 & 1.73 \\
\hline$q=m_{2} / m_{1}$ & \multicolumn{2}{|c|}{0.595} & \multicolumn{2}{|c|}{0.655} \\
\hline Spectral types & O7 III(f) & $\mathrm{O} 8.5 \mathrm{I}$ & & \\
\hline
\end{tabular}

$36 R_{\odot}$ and $29 R_{\odot}$ for the primary and secondary respectively. According to Howarth \& Prinja (1989), the typical radii of $09.7 \mathrm{I}$ and $\mathrm{O} 7.5 \mathrm{I}$ supergiants are 21 and $26 R_{\odot}$ respectively. With that inclination, it seems a priori unlikely that any of the two components fills its Roche lobe. However, we will come back to this point in Sect.7.1.

\section{Spectroscopic luminosity ratio}

In principle, a rough estimate of the visible luminosity ratio of the binary components can be inferred from the ratio of the equivalent widths of absorption lines in the primary's and secondary's spectra (e.g. Rauw et al. 2000a):

$\frac{I_{\mathrm{prim}}}{I_{\mathrm{sec}}}=\left(\frac{E W_{\mathrm{prim}}}{E W_{\mathrm{sec}}}\right)_{\mathrm{obs}} \times\left(\frac{E W_{\mathrm{O} 9.5 \mathrm{I}}}{E W_{\mathrm{O} 7.5 \mathrm{I}}}\right)_{\mathrm{typ}}$

where $\left(\frac{E W_{O 9.5 \mathrm{I}}}{E W_{\mathrm{O} .5 \mathrm{I}}}\right)_{\text {typ }}$ stands for the ratio of the $E W \mathrm{~s}$ of the lines as observed in typical single O9.5 I (as a best substitute for O9.7 I) and O7.5 I stars. We measured the $E W$ ratios of the He I $\lambda \lambda 4026,4471$, He II $\lambda 4542$ and Si IV $\lambda \lambda 4089,4116$ absorption lines (see Table 5 ). The typical $E W$ ratios were derived from the compilation of O-star equivalent widths published by Conti \& Alschuler (1971) and Conti $(1973,1974)$.

In the case of HD 149404, the main limitation of the method stems from the strong orbital variability of the line strength (see Table 5). For instance, the strength of

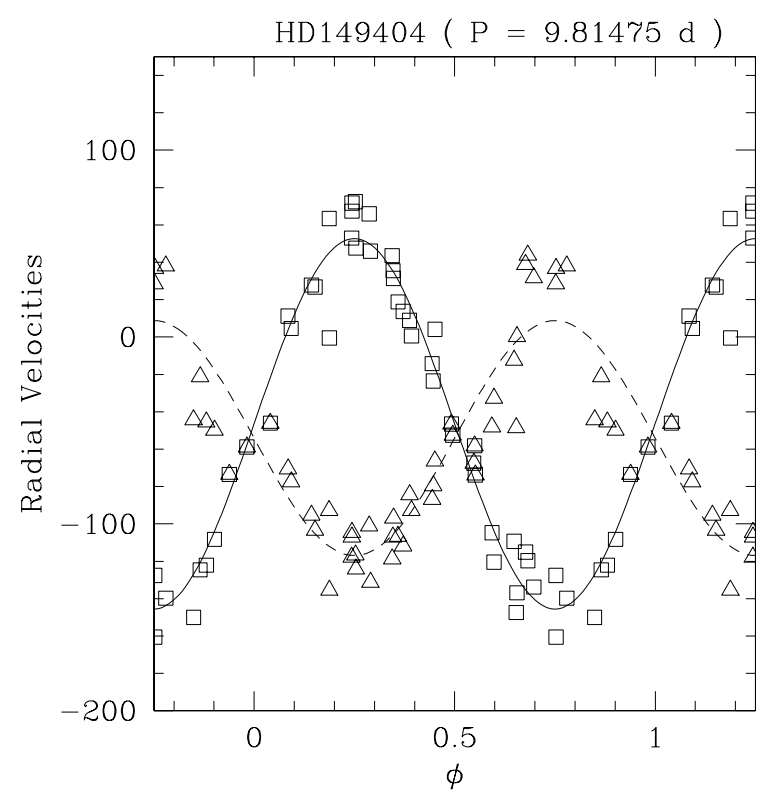

Fig. 4. Radial velocity curve of the He I $\lambda 4471$ line in the spectrum of HD 149404 as a function of the orbital phase and assuming $e=0.0$ (see Table 3 ). The triangles correspond to the primary's $R V \mathrm{~s}$ while the squares indicate the $R V \mathrm{~s}$ of the secondary

the primary's Si IV lines around $\phi \sim 0.75$ is reduced by a factor 2 with respect to $\phi \sim 0.25$, while the strength of the secondary's Si IV lines simultaneously increases by about a factor 1.3. An easy way to account for the fading of the primary's lines around $\phi \sim 0.75$ and the simultaneous strengthening of the secondary's absorptions would be to assume that one of the two stars was brighter on the front side than on the rear. However, since the amplitude of the photometric variations is only a few percent, it seems rather unlikely that the observed $E W$ variations reflect actual variations of the brightness of one of the binary components, otherwise a larger amplitude of the light curve would be expected.

We notice that the total $E W$ (primary + secondary) varies by only about $5 \%$ for the absorptions considered here. On the other side, we know that the He I lines are most probably affected by an emission component and the $E W$ of the primary's He I lines are reduced around 


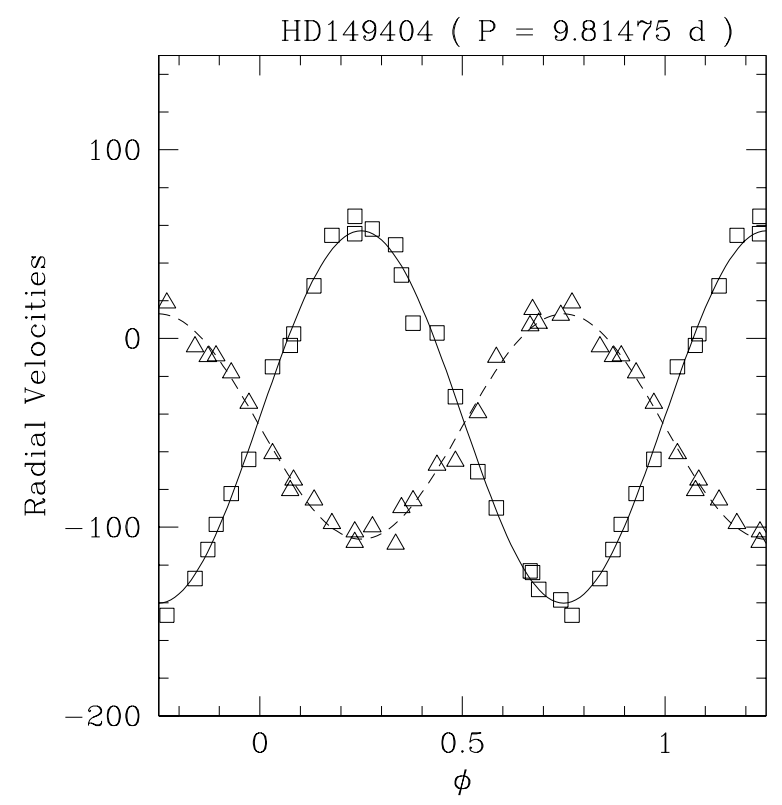

Fig. 5. Radial velocity curve determined by the mask crosscorrelation method as a function of the orbital phase (assuming a circular orbit, see Table 3 )

$\phi \sim 0.75$ (see Fig. 2). Therefore, blending with a most probably slightly red-shifted emission component not associated with either of the two stars could be an explanation for the observed variations.

Similar difficulties are encountered in the UV. Howarth et al. (1997) derived a mean raw UV magnitude difference of 0.8 with the secondary being the brighter component. Correcting this magnitude difference for the different spectral types, we obtain a UV luminosity ratio (primary/secondary) of roughly 0.6. Stickland \& Koch (1996) reported a luminosity ratio of about 1.0 when the primary is moving towards us (our phase 0.25) and about 0.5 when it is moving away (our phase 0.75 ). Using the results for the He II and Si IV lines from Table 5, we calculate a mean luminosity ratio of about $1.4 \pm 0.4$ near $\phi \sim 0.25$ and $0.5 \pm 0.1$ near $\phi \sim 0.75$.

Assuming that the observed $E W \mathrm{~s}$ are indeed reduced by a red-shifted emission component, we can derive the "actual" spectroscopic luminosity ratio using the mean EWs of each star's line when the star is moving towards us. In this way, we find an average ratio of $\bar{I}_{\text {prim }}(0.25) / \bar{I}_{\text {sec }}(0.75)=0.90 \pm 0.16$ (see Table 5$)$.

Alternatively, the absorption enhancement seen when the lines are blueshifted could be due to material from a wind interaction region that would be swept up by the star during its orbital motion. In this case, the front sides of the stars would display abnormal line strengths and the actual luminosity ratio would best be derived from the mean $E W$ s of the lines observed when the stars are moving away from us. This assumption yields an average ratio of $\bar{I}_{\text {prim }}(0.75) / \bar{I}_{\text {sec }}(0.25)=0.75 \pm 0.23$ (see Table 5$)$. This result overlaps within the errors with the value of the $\bar{I}_{\text {prim }}(0.25) / \bar{I}_{\text {sec }}(0.75)$ ratio. In this second scenario, each star would have to sweep up about the same amount of material in order to explain the roughly constant total $E W$ s of the lines. Therefore, it seems more likely that the variations of the apparent luminosity ratio are rather due to an unresolved emission component and in the following we shall thus adopt a spectroscopic luminosity ratio of $0.90 \pm 0.16$.

\section{Emission lines}

The spectrum of HD 149404 exhibits many emission lines some of which display strong orbital variability.

- The unidentified $\lambda \lambda 4486,4504$ emission lines appear to be associated with the secondary star as shown by Nazé et al. (2000). These emission lines are a common feature in the spectra of the most luminous stars between spectral type O7 and O9.5 (Conti 1973; Walborn \& Fitzpatrick 1990);

- N III $\lambda \lambda 4634-41$ : the bulk of the emission seems to follow the primary whilst the lines are observed in absorption in the secondary's spectrum. The $R V$ behaviour of the N III emissions itself is however rather complex and the lines are quite broad and display strong profile variability probably indicating that these lines are not purely stellar features;

- He II $\lambda 4686$ (see Fig. 3) consists of a mixture of emission and absorption lines and the line morphology displays strong phase-locked variations. The emissions do not follow the orbital motion of the stars. Instead, both stars exhibit this line in weak absorption though the visibility of these absorption features varies strongly with phase and the absorption lines vanish near phases 0.2 and 0.7 ;

- A similar situation is observed for the $\mathrm{H} \beta$ line. As for He II $\lambda 4686$, the stellar $\mathrm{H} \beta$ lines are again in (rather weak) absorption and the composite line profile suffers strong variability with an emission feature that does not follow the motion of either star. We caution that the $\mathrm{H} \beta$ profile is blended with the N III $\lambda \lambda 4858-67$ absorption lines and this blend probably affects the radial velocity measurements of the $\mathrm{H} \beta$ emission components;

- Our echelle spectra reveal N II emission features at $\lambda \lambda 5001-12,5667,5932,5940-42,5952,6482$ and 6611 , that are associated with the O9.7 secondary. Walborn (1980) reported weak N II $\lambda \lambda 5940-42,6482$ emission in the ON9.7 Iae star HD 105056. The presence of the N II emissions and the strength of the N III absorption lines in the spectrum of the secondary of HD 149404 could therefore indicate that nitrogen is enhanced in the atmosphere of this star. Further support for an ON classification of the secondary comes from the relative weakness of the C III $\lambda \lambda 4647-50$ absorption compared to the intensity of this line in "normal" O9.7 supergiants (see Walborn \& Fitzpatrick 1990); 
Table 5. Luminosity ratios (primary/secondary) derived for different lines at different orbital phases. Typical uncertainties on the observed $E W$ ratios are less than $10 \%$. The colons indicate those measurements that have larger uncertainties due to heavier blending. The last two columns list the luminosity ratios derived from the mean $E W$ s measured when the components are moving towards us (Col. 7) or when they are moving away from us (Col. 8); see the text for further details

\begin{tabular}{|c|c|c|c|c|c|c|c|}
\hline \multirow{2}{*}{$\begin{array}{l}\text { HJD-2450000 } \\
\phi \\
\end{array}$} & 1299.800 & 1584.857 & 1672.766 & 1304.796 & 1579.879 & \multirow[b]{2}{*}{$\bar{I}_{\text {prim }}(0.25) / \bar{I}_{\text {sec }}(0.75)$} & \multirow[b]{2}{*}{$\bar{I}_{\text {prim }}(0.75) / \bar{I}_{\mathrm{sec}}(0.25)$} \\
\hline & 0.234 & 0.278 & 0.235 & 0.743 & 0.771 & & \\
\hline Не г $\lambda 4026$ & 1.70 & 1.13 & 1.95 & 0.28 & 0.41 & 0.86 & 0.66 \\
\hline Не г $\lambda 4471$ & 1.35 & 1.19 & 1.51 & 0.35 & 0.47 & 0.84 & 0.60 \\
\hline Не II $\lambda 4542$ & 1.71 & 1.22: & 1.95 & & 1.15: & 1.19 & 0.66 \\
\hline Si IV $\lambda 4089$ & 1.07 & 0.90 & 1.11 & 0.39 & 0.91: & 0.83 & 0.61 \\
\hline Si IV $\lambda 4116$ & 1.40 & 1.25 & 1.57 & 0.54 & & 0.80 & 1.21 \\
\hline
\end{tabular}

- The C III $\lambda 5696$ emission is probably formed by two independent features. The strongest component follows the orbital motion of the primary. This trend is confirmed by the variations of the line peak position (see Nazé et al. 2000). At some orbital phases (e.g. $\phi=0.67)$, a second emission peak appears with a velocity indicating that it is most probably associated with the secondary;

- In addition to the absorption lines, He I $\lambda 5876$ displays a variable emission which is particularly strong at phases near 0.0 and 0.5 (see Fig. 3). This emission is probably present in other He I lines too, maybe causing part of the intensity and $E W$ variations;

- The strongest emission feature in the visible spectrum of HD 149404 is $\mathrm{H} \alpha$ (see Fig. 3). The line exhibits a double peaked structure at certain phases, more or less in quadrature with the orbital motion. Though the visibility of the emission peaks in the $\mathrm{H} \beta$ profile is hampered by the blending with the stellar absorption lines, they seem nevertheless to follow more or less the behaviour of the $\mathrm{H} \alpha$ peaks, pointing towards a similar origin. In Sect. 7.2, we will discuss the variability of the $\mathrm{H} \alpha$ emission line in terms of a wind interaction model.

To further analyse the emission features, we apply a socalled "S-wave analysis" (see e.g. Richards et al. 1996). We adopt two axes centred on the center of mass of the binary; the $x$ axis points towards the secondary star while the direction of the $y$ axis is given by the orbital motion of the secondary. We measured the $R V \mathrm{~s}$ of the peaks of the most prominent emission lines and fitted a sine-wave expression

$v(\phi)=-v_{x} \cos (2 \pi \phi)+v_{y} \sin (2 \pi \phi)+v_{z}$

to these points (see e.g. Rauw et al. 1999). The results are summarized in Table 6 and a projected Doppler map is presented in Fig. 6. We caution that the quality of the fit to the $R V \mathrm{~s}$ of the $\mathrm{N}$ III $\lambda \lambda 4634-41$ lines is rather poor, mainly because of the variable shape of these lines.

The positions of the $\lambda \lambda 4486,4504, \mathrm{C}$ III $\lambda 5696, \mathrm{~N}_{\text {III }}$ $\lambda \lambda 4634-41$ and NII $\lambda \lambda 5932,5942$ emission regions in velocity space indicate that the bulk of these emissions arises most probably within the atmospheres of the stars.
Table 6. Results of the S-wave analysis: Coralie observations are not included for the N III emissions

\begin{tabular}{lrrr}
\hline & \multicolumn{1}{c}{$v_{x}$} & \multicolumn{1}{c}{$v_{y}$} & \multicolumn{1}{c}{$v_{z}$} \\
\hline \hline$? \lambda 4486$ & -0.1 & 87.3 & -39.1 \\
$? \lambda 4504$ & 2.7 & 80.6 & 6.5 \\
N III $\lambda 4634$ & -9.6 & -73.6 & -36.9 \\
N III $\lambda 4641$ & -16.9 & -105.8 & -52.8 \\
C III $\lambda 5696$ & 22.3 & -62.6 & -42.5 \\
N II $\lambda 5932$ & 8.3 & 108.0 & -81.7 \\
N II $\lambda 5942$ & 16.3 & 109.7 & -44.8 \\
He II $\lambda 4686$ & -128.6 & -82.4 & -99.7 \\
He II $\lambda 4686$ & 80.6 & -65.3 & 16.4 \\
H $\beta$ & -123.8 & -153.2 & -47.1 \\
H $\beta$ & 136.7 & 57.0 & -47.8 \\
H $\alpha$ & -119.2 & -75.1 & -44.4 \\
H $\alpha$ & 106.2 & 24.0 & -53.4 \\
\hline
\end{tabular}

On the other side, our analysis reveals that this is not the case for the $\mathrm{H} \alpha, \mathrm{H} \beta$ and $\mathrm{He}$ II $\lambda 4686$ emissions. The latter lines exhibit a double peaked structure at orbital phases near conjunction and a single peak around quadrature. Assuming that the two peaks actually cross over, we have fitted their $R V \mathrm{~s}$ with the above sine-wave expression. We caution here that only the $\mathrm{H} \alpha$ peaks are free from blends with absorption lines. The $\mathrm{H} \beta$ and He II $\lambda 4686$ lines are usually "polluted" by absorption lines and the results for these lines should therefore be regarded only as trends roughly confirming the behaviour of the $\mathrm{H} \alpha$ peaks.

\section{Discussion}

\subsection{The evolutionary status}

Adopting the effective temperature calibration of Chlebowski \& Garmany (1991), the spectral types we have derived yield $T_{\text {eff }}=35100 \pm 1000 \mathrm{~K}$ and $T_{\text {eff }}=$ $30500 \pm 400 \mathrm{~K}$ for the primary and the secondary respectively. HD 149404 has an apparent $V$ magnitude of 5.47 (Drilling 1991). Assuming that the star is a member of the Ara OB1a association ( $D M=10.7$, Humphreys 1978) and adopting a colour excess $E(B-V)=0.68$ (Shull \& Van Steenberg 1985) and a spectroscopic luminosity 


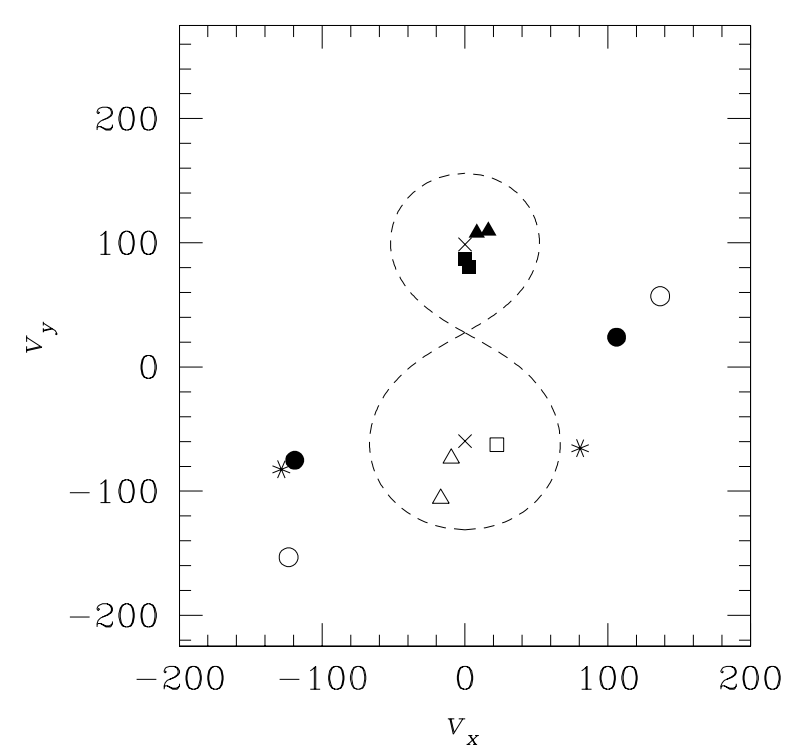

Fig. 6. Doppler map showing the results of our S-wave analysis projected on the $\left(v_{x}, v_{y}\right)$ velocity plane. The crosses indicate the velocity of the center of mass of the binary components as derived from our orbital solution while the dashed line is the equivalent of the Roche lobe in velocity space. The different symbols stand for different emission lines: filled circles $=\mathrm{H} \alpha$; open circles $=\mathrm{H} \beta$; filled triangles $=\mathrm{N}$ II $\lambda \lambda 5932,5942$; open triangles $=\mathrm{N}$ III $\quad \lambda \lambda 4634-41 ;$ filled squares $=\lambda \lambda 4486,4504 ;$ open square $=$ C III $\lambda 5696$ and stars $=$ He II $\lambda 4686$

ratio of $0.90 \pm 0.16$ (see above), we derive $\log \left(L_{\mathrm{bol}}^{\mathrm{prim}} / L_{\odot}\right)=$ $5.90 \pm 0.08$ and $\log \left(L_{\mathrm{bol}}^{\mathrm{sec}} / L_{\odot}\right)=5.78 \pm 0.08$ for the primary and secondary respectively. We caution that the error bars on the luminosities correspond only to the estimated error on the luminosity ratio and an uncertainty of half a spectral class for the bolometric correction. Our error bars account by no means for the (probably large) uncertainties on the distance of HD 149404.

The resulting locations of the components of HD 149404 in a H-R diagram are shown in Fig. 7 together with the theoretical evolutionary tracks of Schaller et al. (1992). We notice that the secondary star (i.e. the least massive star) seems to be the most evolved component though it lies on an evolutionary track of lower initial mass than the primary. This was already found by Penny et al. (1996), though these authors adopted different spectral types and hence derived different positions for the stars in the H-R diagram. As pointed out in Sect. 6, the strong N III absorptions and the N II emission lines seen in the secondary's spectrum could indicate that nitrogen is slightly enhanced in the atmosphere of this star, which would be a further indication that this star must be an evolved object. A possibility to account for the evolutionary status of the secondary would be to suppose that this star was initially the more massive component of the binary and has lost (or transferred) a considerable fraction of its mass through Roche lobe overflow in the past (see also Penny et al. 1996).

The temperatures and luminosities derived here correspond to radii of 24.3 and $28.1 R_{\odot}$ for the primary and the

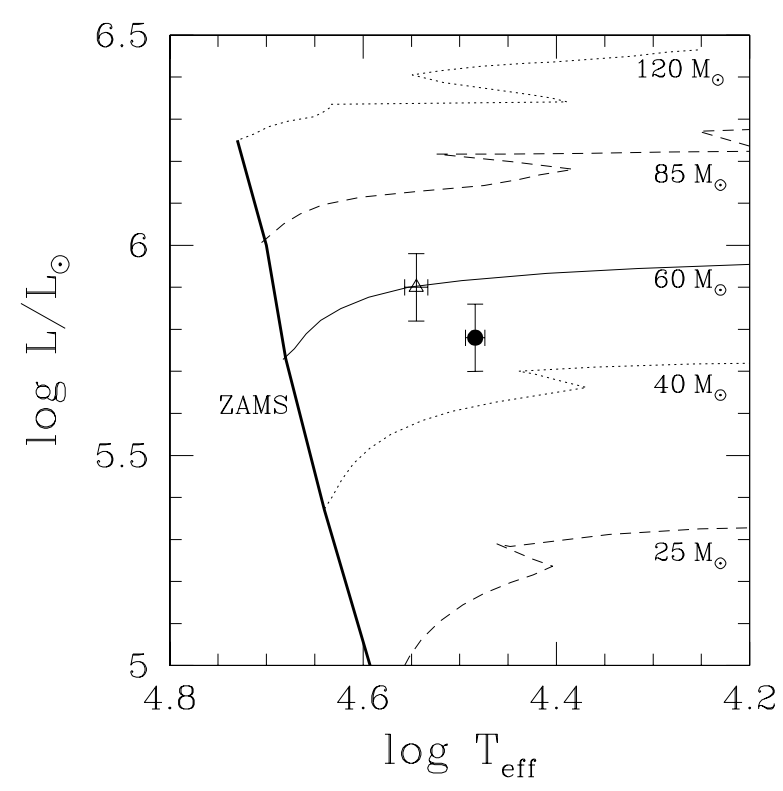

Fig. 7. Hertzsprung-Russell diagram of HD 149404. The open triangle stands for the primary star whilst the filled circle yields the position of the secondary star

secondary respectively. Therefore, the radius of the secondary star appears larger than we would expect from the "typical" parameters listed by Howarth \& Prinja (1989). Howarth et al. (1997) derived projected rotational velocities of $v_{\mathrm{e}} \sin i=91 \mathrm{~km} \mathrm{~s}^{-1}$ and $100 \mathrm{~km} \mathrm{~s}^{-1}$ for the 07.5 and the O9.7 component respectively. Assuming that the stars have the same angular rotational velocity (though the rotation needs not necessarily be synchronous with the orbital motion), we can derive the ratio of the stellar radii from the ratio of the projected rotational velocities. The value obtained in this way $R_{\text {prim }} / R_{\text {sec }}=0.91$ is in very good agreement with the ratio of the radii derived above (0.86).

At this point, we need to return to the question of a current RLOF. In fact, the radius of the secondary star derived hereabove ${ }^{3}\left(28.1 R_{\odot}\right)$ is only slightly smaller than the radius of its Roche lobe $\left(29 R_{\odot}\right)$ for an inclination of $21^{\circ}$. Therefore a small error on the (poorly constrained) inclination has a large impact on our conclusion about the current configuration of HD 149404. Indeed, it could be that the secondary has just finished RLOF and is now settling down on a "normal" evolutionary track corresponding to its current mass. Alternatively, the secondary could still be filling its critical volume and could be suffering an enhanced mass loss through the inner Lagrangian point.

\subsection{Towards a model for the wind interaction}

In Sect. 6 we have shown that the $\mathrm{H} \alpha, \mathrm{H} \beta$ and $\mathrm{He}$ II $\lambda 4686$ emission lines are not formed in the atmospheres of the stars but must be formed somewhere between the stars.

\footnotetext{
${ }^{3}$ We caution that the actual value of $R_{\mathrm{sec}}$ depends on the adopted luminosity ratio between the two stars and the adopted distance towards HD 149404.
} 


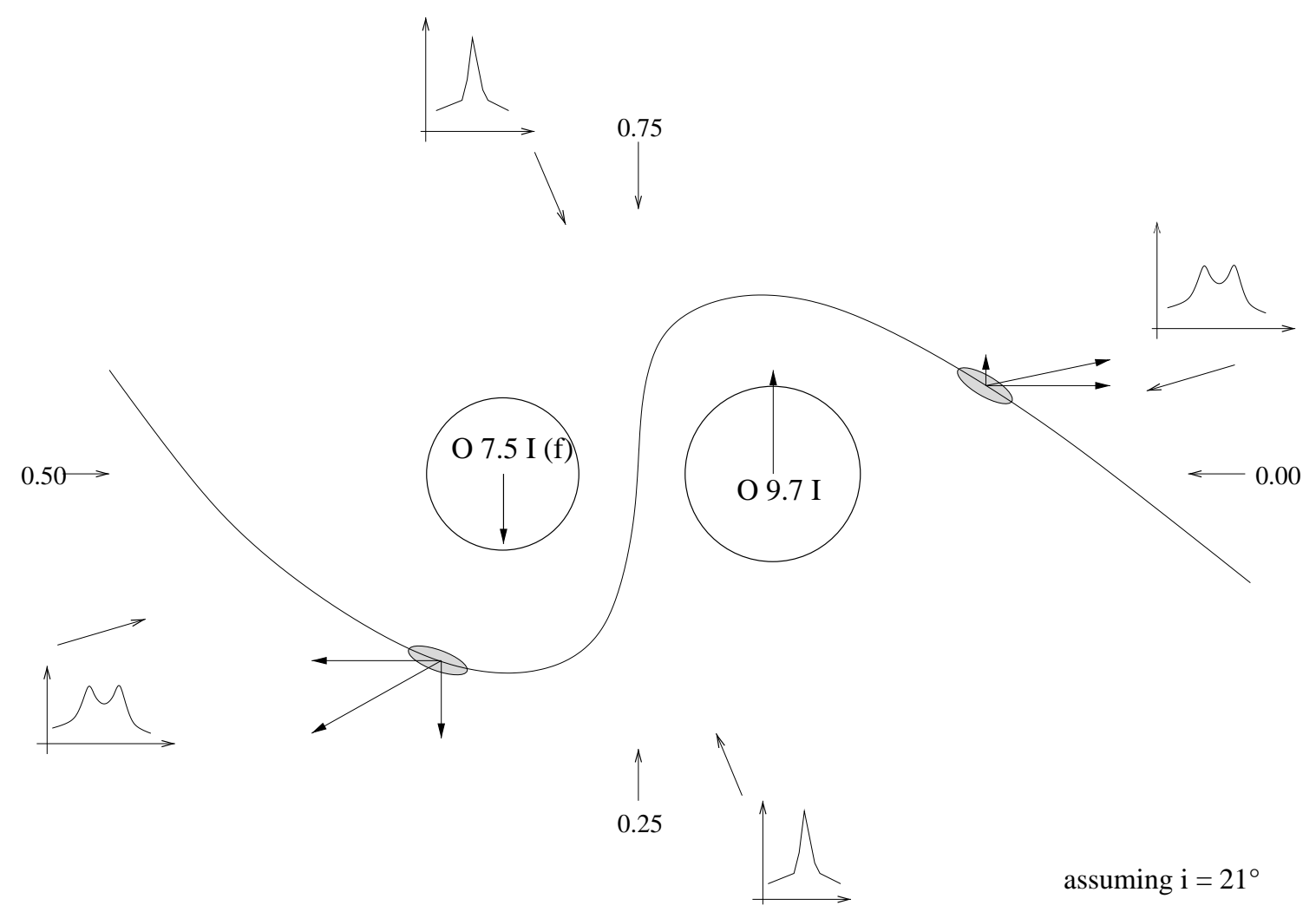

Fig. 8. Schematic view of the wind interaction in the HD 149404 system projected on the orbital plane. The changing morphology of the $\mathrm{H} \alpha$ line at some specific orbital phases is also sketched as a function of the line of sight. The relative dimensions of the orbit and the stars correspond to an inclination of $i=21^{\circ}$

Thaller (1998) suggested that the $\mathrm{H} \alpha$ emissions were coming from two focused stellar winds flowing towards a colliding wind interaction between the stars. Though the radius of the secondary star derived in Sect.7.1 supports the possibility of an enhanced mass loss from the secondary focused towards the primary, it is not clear how the model suggested by Thaller (1998) could account for the $R V$ behaviour found in Sect. 6 . We favour an alternative model where the $\mathrm{H} \alpha$ emissions arise in the arms of a colliding wind shock region. Figure 8 displays a schematic view of such a wind interaction. As pointed out above, the secondary might actually be suffering a stronger mass loss than would be expected for a "normal" O9.7 I star. Therefore, we assume that the two stars have roughly equally strong winds. The Coriolis force bends the arms of the shock region around both stars. In this model, the $\mathrm{H} \alpha$ emission forms through recombination in the hatched areas where the density is enhanced due to the shock (Fig. 8). The arrows indicate the velocity components of the $\mathrm{H} \alpha$ emission regions as inferred from our S-wave analysis. The velocity components result from the combination of the orbital motion and the flow velocity of the plasma in the shock region.

In Fig. 8, the projections of the line of sight on the plane of the orbit are indicated for orbital phases near conjunction and quadrature. We see that this rather simple model can at least qualitatively account for the changing morphology and the $R V$ behaviour of the $\mathrm{H} \alpha$ emission line in the spectrum of HD 149404.

We have analyzed ROSAT observations of HD 149404 to search for a signature of the wind interaction in the X-ray domain. HD 149404 was observed twice with ROSAT. A first exposure was obtained as part of the ROSAT All Sky Survey (Berghöfer et al. 1996). Another $3 \mathrm{ksec}$ PSPC exposure was obtained on JD 2449056.813 $(\phi=0.70)$. We have retrieved this latter observation (rp201266) from the ARNIE database at Leicester University. The data were reduced using the XSELECT and XSPEC softwares. The background corrected PSPC count rate is $8.010^{-2} \mathrm{cts} \mathrm{s}^{-1}$. The PSPC spectrum is quite hard, as the ratio of the count rates in the ranges $0.5-2 \mathrm{keV}$ and $0.1-0.5 \mathrm{keV}$ is about 25. Despite the limited $S / N$ ratio, we could fit an absorbed Raymond-Smith model (Raymond \& Smith 1977) to the PSPC spectrum fixing the neutral hydrogen column density to its interstellar value $N_{\mathrm{H}}^{\mathrm{ISM}}=2.510^{21} \mathrm{~cm}^{-2}$ (Shull \& Van Steenberg 1985). The best fit yields an X-ray temperature of $0.84 \pm$ $0.10 \mathrm{keV}$ and a luminosity in the $0.1-2.0 \mathrm{keV}$ energy range of $L_{\mathrm{X}}=1.610^{32} \mathrm{erg} \mathrm{s}^{-1}$ corresponding to a ratio $L_{\mathrm{X}} / L_{\mathrm{bol}}$ of $3.010^{-8}$, slightly lower than for typical $\mathrm{O}$ stars. This result is in agreement with the value found by Berghöfer et al. (1996). Thus the snapshot ROSAT observations reveal no indication of a strong X-ray excess that could be attributed to a colliding wind emission. This is in line with 
the results of Chlebowski \& Garmany (1991) who found no significant X-ray excess in those O-star binaries that are in a semi-detached or a contact configuration.

\subsection{Summary and conclusions}

We have used an extensive set of high resolution spectra to derive new orbital elements for the early-type binary HD 149404. We have shown that the mask crosscorrelation technique developed at the Geneva observatory can be successfully applied to disentangle heavily blended lines in massive binaries. Our results allowed us to propose a new spectral classification for the components. We find that the primary is of spectral type O7.5I(f), while the secondary is most likely an ON9.7 I supergiant. The secondary seems to be the most evolved component of the system and its current evolutionary status could best be explained if the system has undergone a Roche lobe overflow episode during the past. The secondary could actually still be rather close to filling its critical volume and this could lead to an enhanced mass loss of the secondary.

The behaviour of the emission lines in the spectrum of HD 149404 points towards a wind interaction between the two stars. We propose a simple model where some of the optical emission lines arise in a heavily bended shock region. It seems likely that HD 149404 is currently in a rather short-lived evolutionary stage where the wind interaction could be strongly affected by an enhanced mass loss of the secondary star. We caution, however, that this rather simple picture of the interaction could be seriously complicated if the secondary actually filled its critical volume. In this context, we emphasize that some of the crucial parameters of HD 149404 such as its distance and the orbital inclination remain poorly constrained. All future observations that could help to determine these parameters would be extremely useful to fully understand this system.

Acknowledgements. We are grateful to H. Sana for taking the May 2000 spectra and for his help in the reduction of the FEROS data, and to Dr. E. Jehin and G. Parmentier for taking some of the CAT + VLC observations. We also thank an anonymous referee for his helpful comments. We are greatly indebted to the Fonds National de la Recherche Scientifique for multiple support. This research is also largely supported by contract P4/05 "Pôle d'Attraction Interuniversitaire" (SSTC-Belgium) and through the PRODEX XMM-OM and Integral Projects. Part of this work has been supported by the Swiss National Found for the Scientific Research. The SIMBAD database has been consulted for the bibliography.

\section{References}

Baranne, A., Queloz, D., Mayor, M., et al. 1996, A\&AS, 119, 373

Berghöfer, T. W., Schmitt, J. H. M. M., \& Cassinelli, J. P. 1996, A\&AS, 118, 481

Chlebowski, T., \& Garmany, C. D. 1991, ApJ, 368, 241

Conti, P. S. 1973, ApJ, 179, 181

Conti, P. S. 1974, ApJ, 187, 539
Conti, P. S., \& Alschuler, W. R. 1971, ApJ, 170, 325

Conti, P. S., \& Frost, S. A. 1977, ApJ, 212, 728

Conti, P. S., Leep, E. M., \& Lorre, J. J. 1977, ApJ, 214, 759

Drilling, J. S. 1991, ApJS, 76, 1033

Gies, D. R., Bagnuolo, W. G. Jr., \& Penny, L. R. 1997, ApJ, 479,408

Heck, A., Manfroid, J., \& Mersch, G. 1985, A\&AS, 59, 63

Howarth, I. D., \& Prinja, R. K. 1989, ApJS, 69, 527

Howarth, I. D., Siebert, K. W., Hussain, G. A. J., \& Prinja, R. K. 1997, MNRAS, 284, 265

Hubeny, I., Lanz, T., \& Jeffery, C. S. 1994, TLUSTY and SYNSPEC: A User's Guide, Newsletter on Analysis of Astronomical Spectra (Univ. of St. Andrews)

Humphreys, R. M. 1978, ApJS, 38, 309

Hutchings, J. B., \& van Heteren, J. 1981, PASP, 93, 626

Kaufer, A., Stahl, O., Tubbesing, S., et al. 1999, The Messenger, 95, 8

Kupka, F., Piskunov, N., Ryabchikova, T. A., Stempels, H. C., \& Weiss, W. W. 1999, A\&AS, 138, 119

Kurucz, R. L. 1994, Solar abundance model atmospheres for 0 , 1, 2, 4, $8 \mathrm{~km} \mathrm{~s}^{-1}$, CD-ROM No. 19, Smith. Astroph. Obs.

Lafler, J., \& Kinman, T. D. 1965, ApJS, 11, 216

Lucy, L. B., \& Sweeney, M. A. 1971, AJ, 76, 544

Luna, H. G. 1988, A\&AS, 74, 427

Massey, P., \& Conti, P. S. 1979, in IAU Symp. 83, Mass Loss and Evolution of O-Type Stars, ed. P. S. Conti, \& C. W. H. de Loore, 271

Mathys, G. 1988, A\&AS, 76, 427

Mathys, G. 1989, A\&AS, 81, 237

Mihalas, D., \& Hummer, D. G. 1973, ApJ, 179, 827

Morrison, N. D. 2000, private communication

Nazé, Y., Carrier, F., \& Rauw, G. 2000, in Interacting Winds from Massive Stars, ed. A. F. J. Moffat, \& N. St.-Louis, ASP Conf. Ser., in press

Penny, L. R., Gies, D. R., \& Bagnuolo, W. G. Jr. 1996, in Proc. 33rd Liège Int. Astroph. Coll., Wolf-Rayet Stars in the Framework of Stellar Evolution, ed. J.-M. Vreux et al., 289

Rauw, G., Vreux, J. M., \& Bohannan, B. 1999, ApJ, 517, 416

Rauw, G., Sana, H., Gosset, E., et al. 2000a, A\&A, 360, 1003

Rauw, G., Sana, H., Vreux, J.-M., Gosset, E., \& Stevens, I. R. 2000b, in Interacting Winds from Massive Stars, ed. A. F. J. Moffat, \& N. St.-Louis, ASP Conf. Ser., in press

Raymond, J. C., \& Smith, B. W. 1977, ApJS, 35, 419

Renson, P. 1978, A\&A, 63, 125

Richards, M. T., Jones, R. D., \& Swain, M. A. 1996, ApJ, 459, 249

Schaller, G., Schaerer, D., Meynet, G., \& Maeder, A. 1992, A\&AS, 96, 269

Shull, J. M., \& Van Steenberg, M. E. 1985, ApJ, 294, 599

Stickland, D. J., \& Koch, R. H. 1996, The Observatory, 116, 145

Stickland, D. J. 1997, The Observatory, 117, 37

Thaller, M. L. 1997, ApJ, 487, 380

Thaller, M. L. 1998, in Proc. Boulder-Munich II Worksh., Properties of Hot, Luminous Stars, ed. I. D. Howarth, ASP Conf. Ser., 131, 417

Vanbeveren, D., \& de Loore, C. 1980, A\&A, 86, 21

Walborn, N. R. 1971, ApJS, 23, 257

Walborn, N. R. 1972, AJ, 77, 312

Walborn, N. R. 1980, ApJS, 44, 535

Walborn, N. R., \& Fitzpatrick, E. L. 1990, PASP, 102, 379

Wiggs, M. S., \& Gies, D. R., 1993, ApJ, 407, 252 